\title{
Silver-Soret Nanoparticles for Femtomolar Sensing of Glutathione in a Surface Plasmon-Coupled Emission Platform
}

\section{Supporting Information}

Seemesh Bhaskar ${ }^{1}$, Maku Moronshing ${ }^{2}$, Venkatesh Srinivasan ${ }^{1}$, Pradeep Kumar Badiya ${ }^{1}$, Chandramouli Subramaniam ${ }^{2 *}$ and Sai Sathish Ramamurthy ${ }^{1 *}$

${ }^{1}$ STAR Laboratory, Department of Chemistry, Sri Sathya Sai Institute of Higher Learning, Prasanthi Nilayam, Puttaparthi 515134, Anantapur, Andhra Pradesh, INDIA.

${ }^{2}$ Department of Chemistry, Indian Institute of Technology Bombay, Powai, Mumbai 400076, Maharashtra, INDIA

\section{AUTHOR INFORMATION}

Corresponding Author

*Corresponding author:

*S.S.R.: Sai Sathish Ramamurthy: Tel - +91-8790314405. Email: rsaisathish@sssihl.edu.in *C.R.: Chandramouli Subramaniam: Tel - +91-9769927194. Email: csubbu@ chem.iitb.ac.in

\section{Chemicals:}

Rhodamine 6G (Rh6G), poly vinyl alcohol (PVA), Reduced L-glutathione (GSH), Alizarin Red S (ARS) were procured from Sigma-Aldrich. 50nm silver thin film coated on pyrex with $5 \mathrm{~nm}$ silica layer on it, were purchased from EMF Corp, USA. 

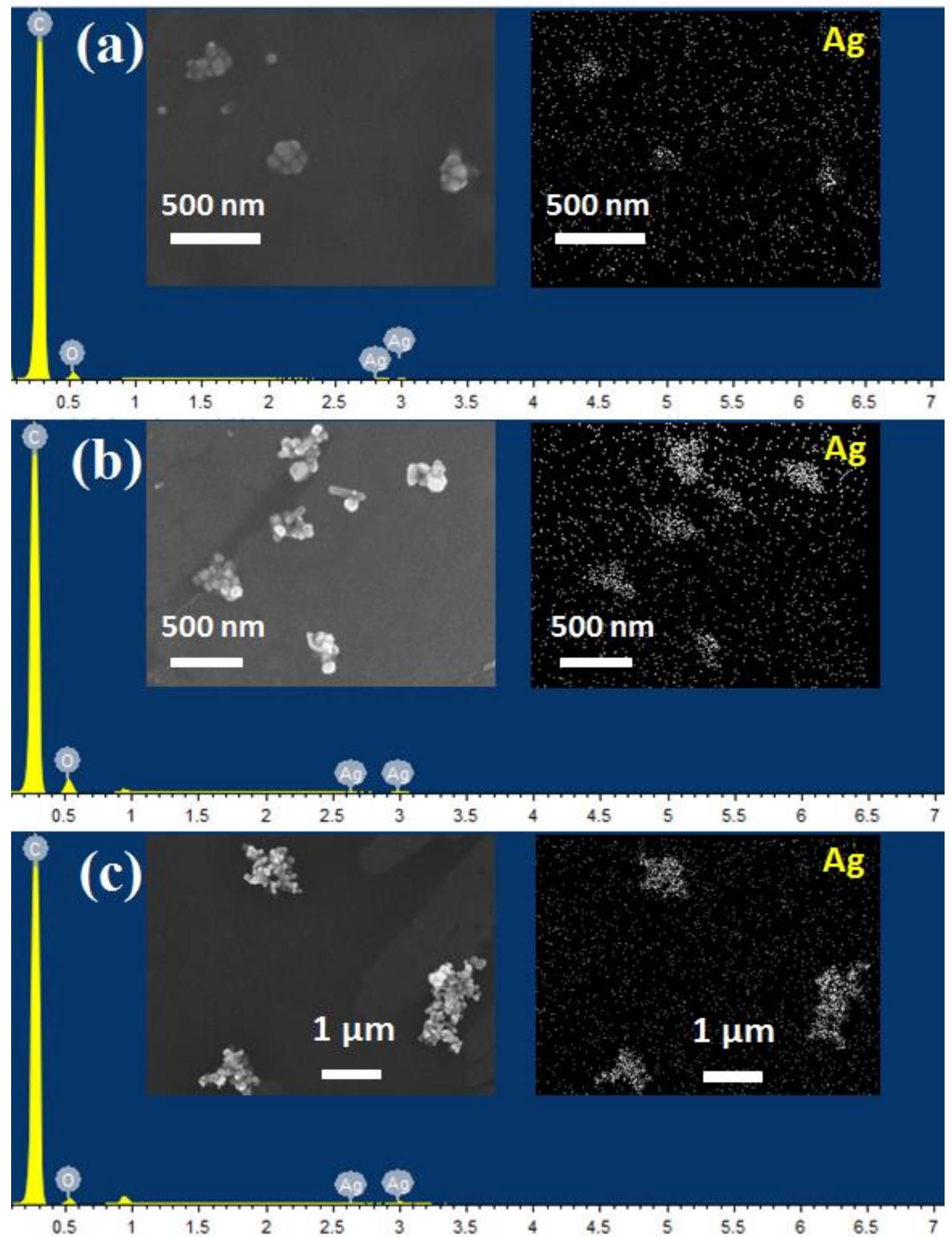

Figure S1. a, b and c depicts the EDAX results for the corresponding SEM images presented as an inset for SC60, SC90 and SC120 doped in PVA. 

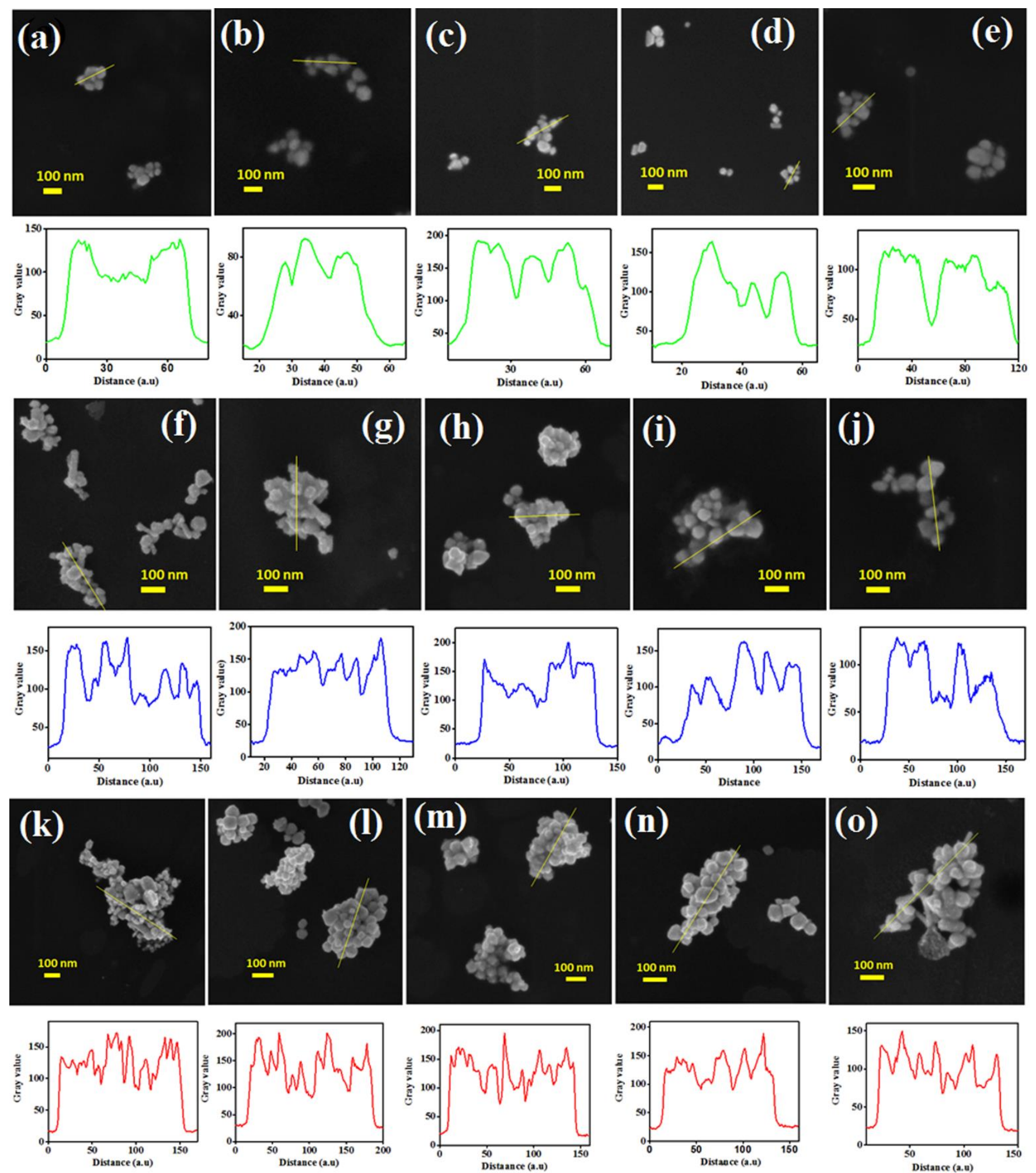

Figure S2. Top figures a, b, c, d, e; middle figures f, g, h, i, j; bottom figures k, 1, m, n, o consist of multiple SEM images of SC60; SC90 and SC120 along with their corresponding height profiles below each of the individual figures. 

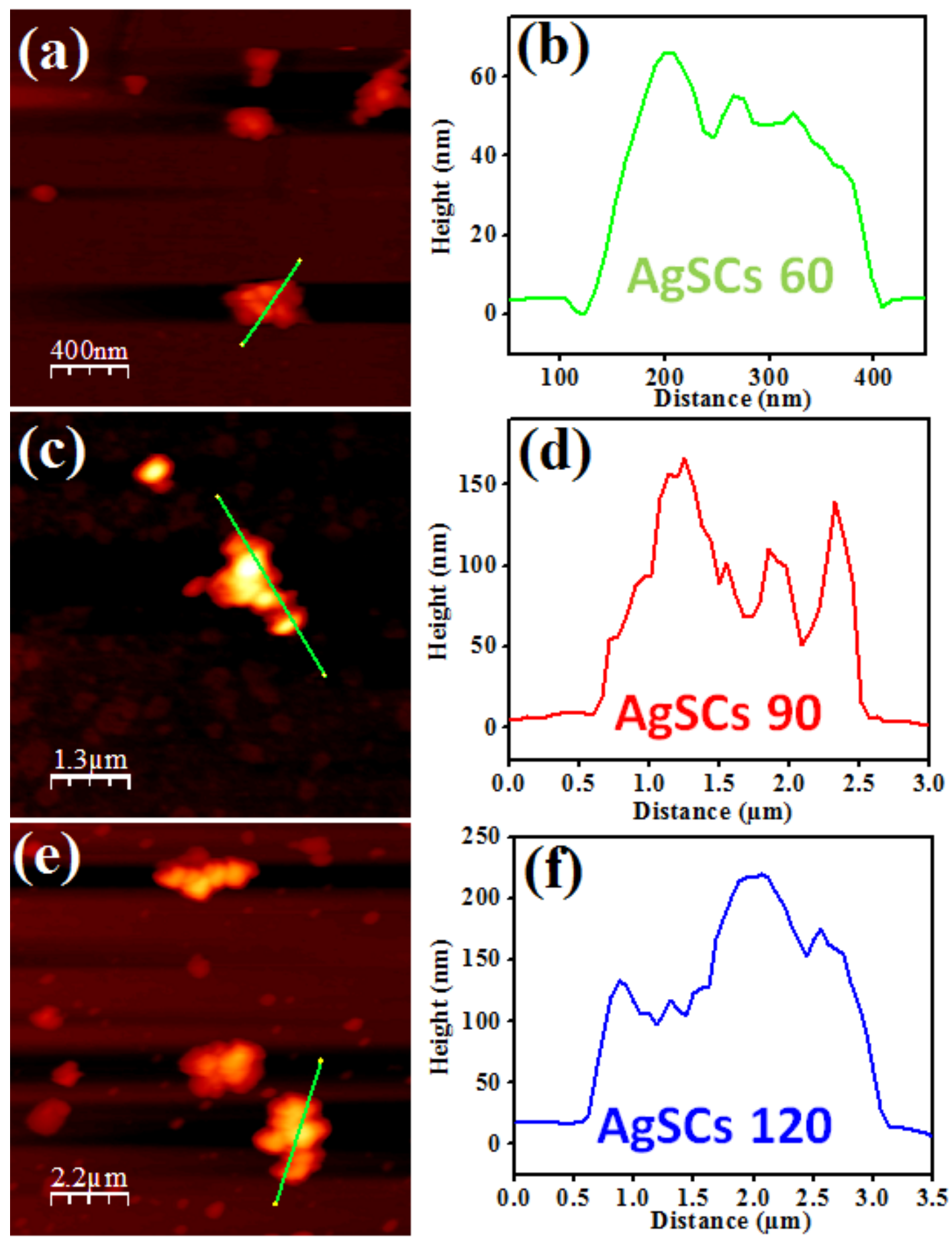

Figure S3. Top figures a, b; middle figures c, d; bottom figures e, f shows AFM images and the corresponding line profiles of SC60; SC90 and SC120. 


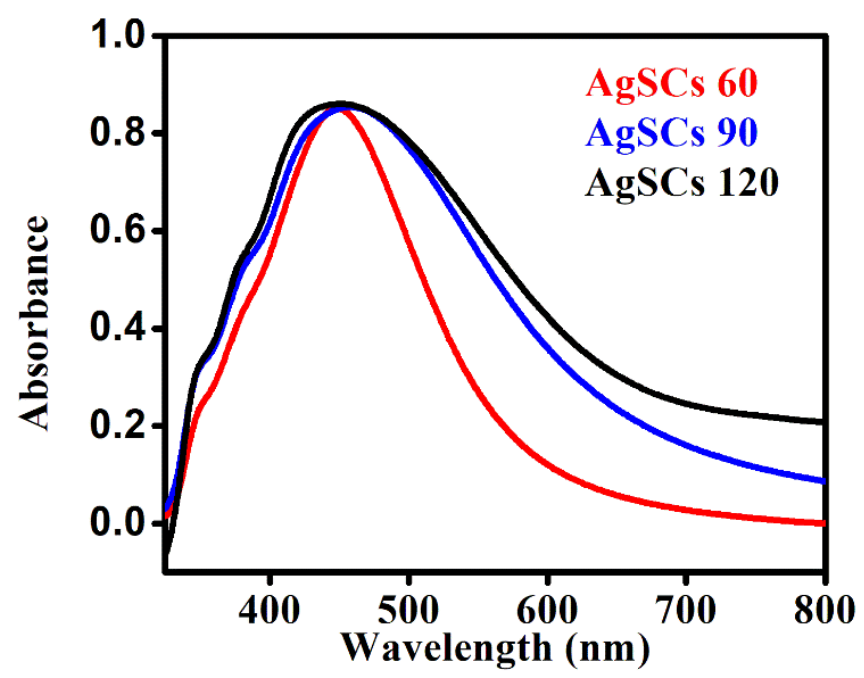

Figure S4. UV-Vis absorbance spectra of AgSCs60, AgSCs90 and AgSCs120.
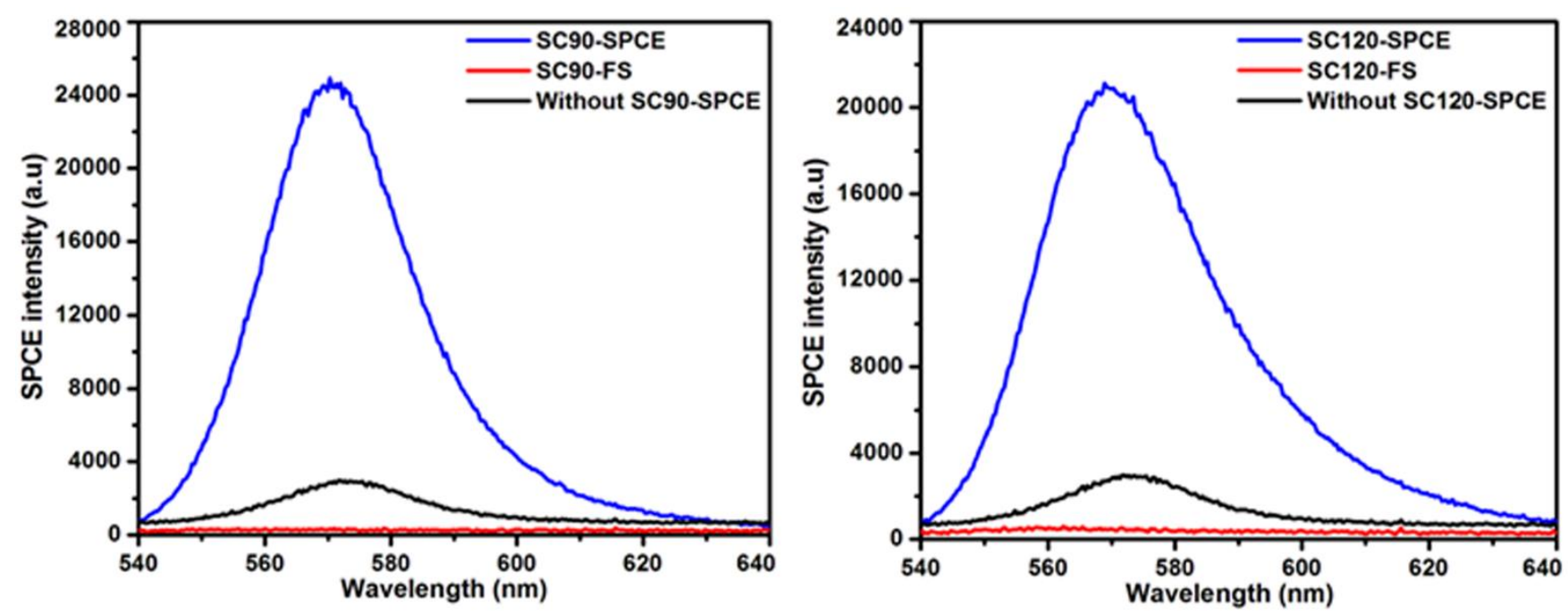

Figure S5. SPCE and FS spectra using SC90, SC120 and blank in spacer architecture. 

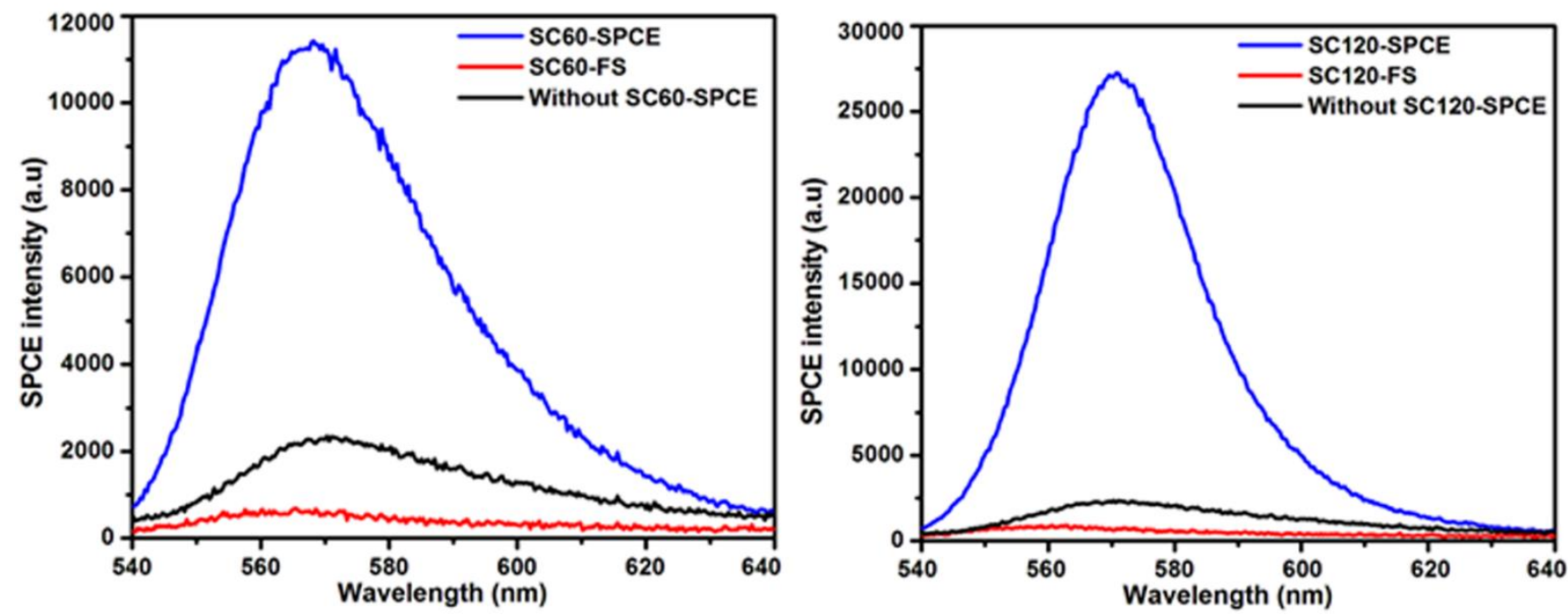

Figure S6. SPCE and FS spectra using Sorets (60 and 120) and blank in cavity architecture.
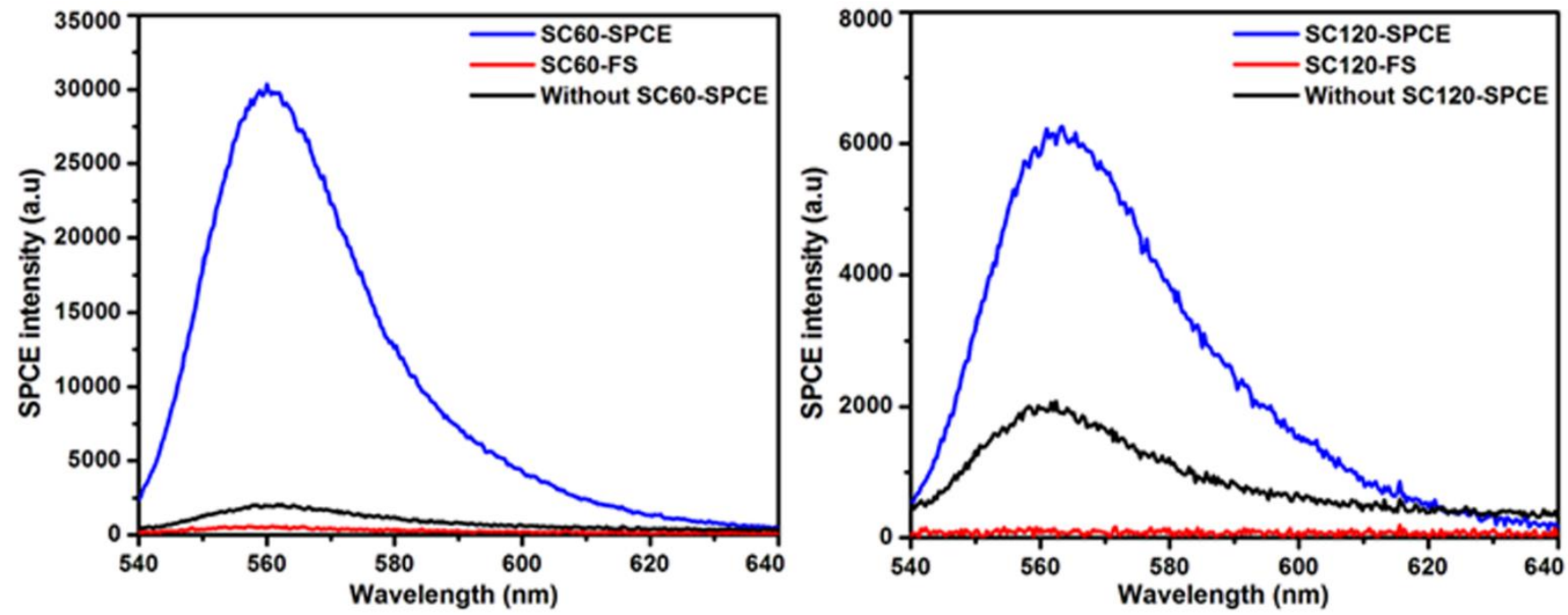

Figure S7. SPCE and FS spectra using SC60, SC120 and blank in pseudo-MDM architecture. 

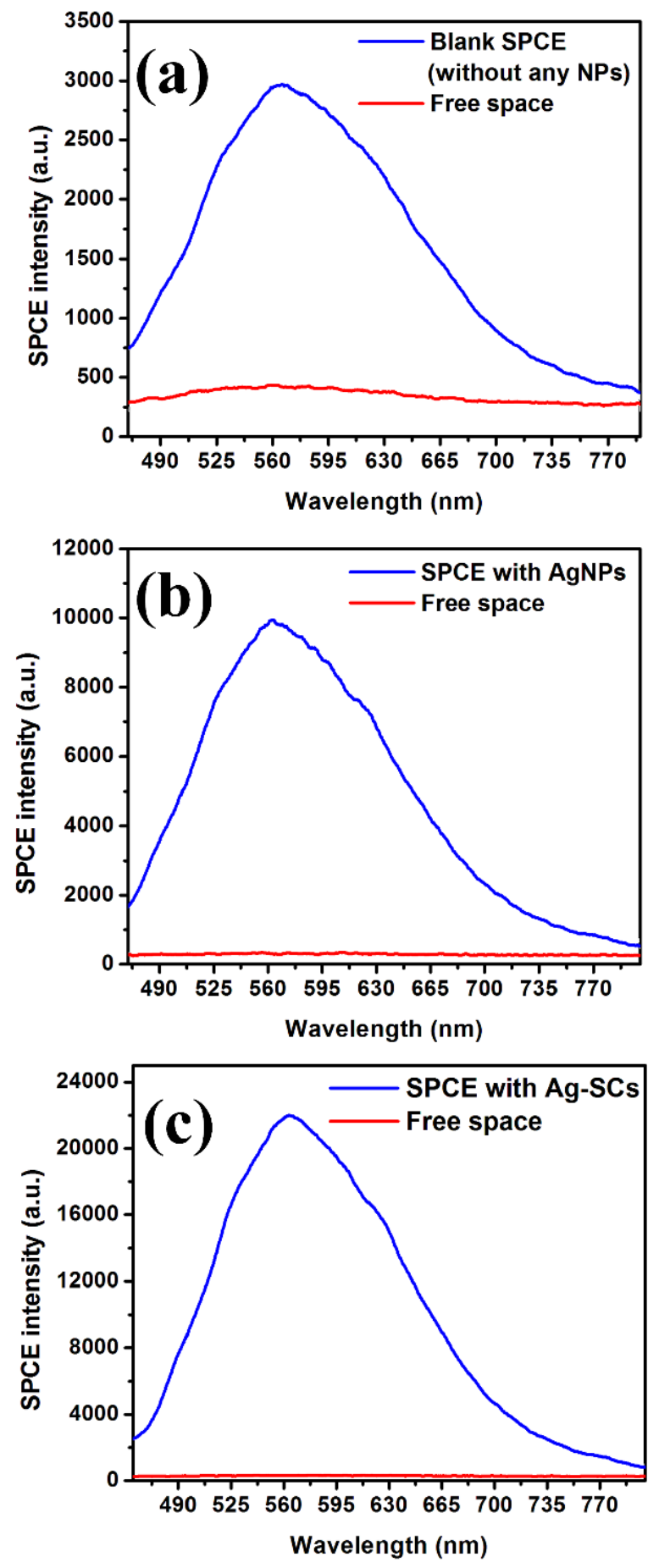

Figure S8. SPCE spectra from ARS using (a) blank (without NPs), (b) only AgNPs (without sorets) and (c) Ag-SCs, as spacer material along with their corresponding FS emission. These plots were used for calculation of corresponding enhancements (SPCE/FS) which gives 7, 46 and 97-fold enhancements for (a), (b) and (c). 
Table S1. Comparison of recent techniques/strategies developed to detect GSH with SPCE platform, presented along with their limit of detection (LOD) achieved.

\begin{tabular}{|l|l|l|l|}
\hline $\begin{array}{l}\text { Sl. } \\
\text { No. }\end{array}$ & Technique/strategy & LOD & Reference \\
\hline 1. & $\begin{array}{l}\text { Förster resonance energy transfer (FRET) phenomena } \\
\text { between MoS } 2 \text { quantum dots QDs and Rh6G molecules }\end{array}$ & $2.7 \mathrm{nM}$ & 39 \\
\hline 2. & $\begin{array}{l}\text { Acetylene black and chitosan composite in a voltam- } \\
\text { metric method }\end{array}$ & $5.8 \times 10^{-8} \mathrm{M}$ & 43 \\
\hline 3. & $\begin{array}{l}\text { Molecule developed such as 6-methoxynaphthalene-2, } \\
\text { 3-dicarbaldehyde (MNDA) with two-photon capability } \\
\text { was adopted for GSH detection using two-photon mi- } \\
\text { croscopy (TPM) }\end{array}$ & $\begin{array}{l}6.4 \times 10^{-8} \mathrm{M} \\
\text { Colorimetric determination based on CuS-polydopa- } \\
\text { mine-Au composite }\end{array}$ & $0.42 \mu \mathrm{M}$ \\
\hline 4. & Different electrochemical methods are reviewed & $1 \mathrm{nM}$ & 42 \\
\hline 6. & $\begin{array}{l}\text { Silver-Soret Nanoparticles for Femtomolar Sensing of } \\
\text { Glutathione in a Surface Plasmon-Coupled Emission } \\
\text { Platform }\end{array}$ & $250 \mathrm{fM}$ & $\begin{array}{l}\text { Current } \\
\text { study }\end{array}$ \\
\hline
\end{tabular}
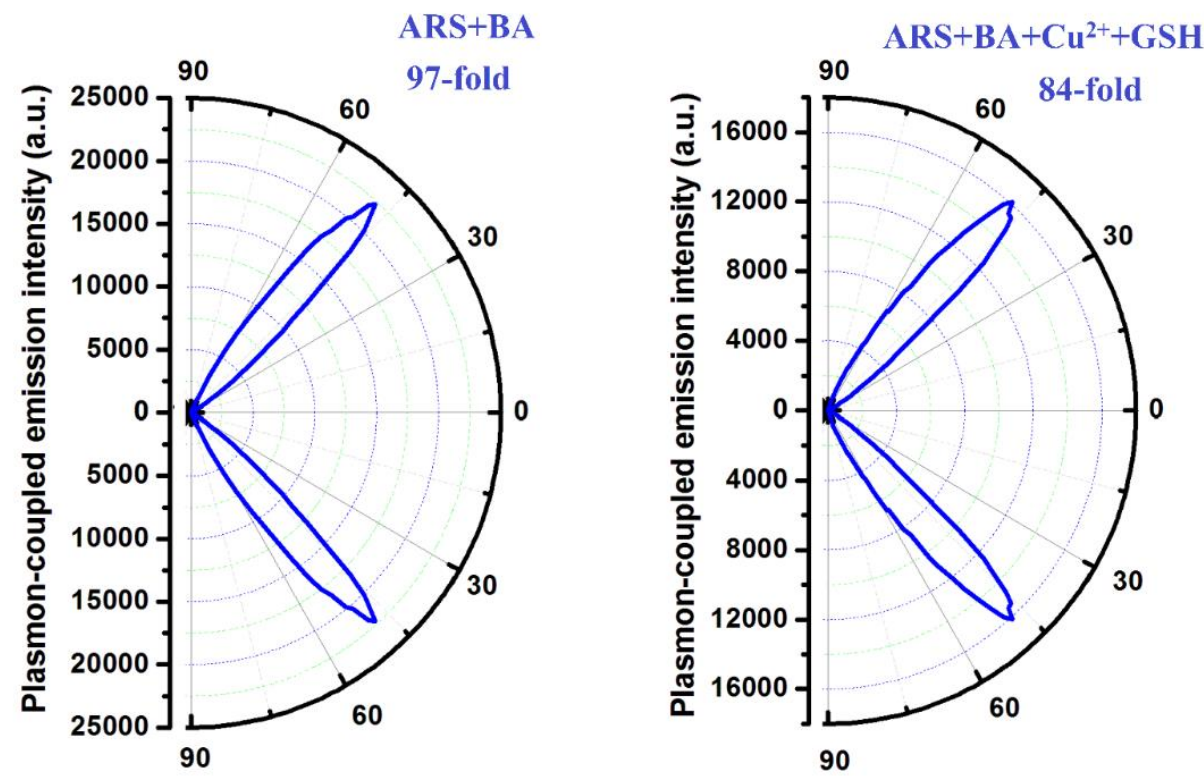

Figure S9. Angularity plot for plasmon-coupled emission from ARS+BA without $\mathrm{Cu}^{2+}$ with 97-fold enhancement [indicating maximum plasmon-coupled emission in the absence of quencher] (left). Angularity

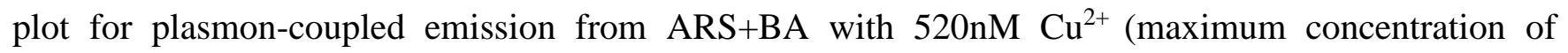
quencher) and $1 \mu \mathrm{M}$ GSH solution [maximum concentration of dequenching moiety] (right). 

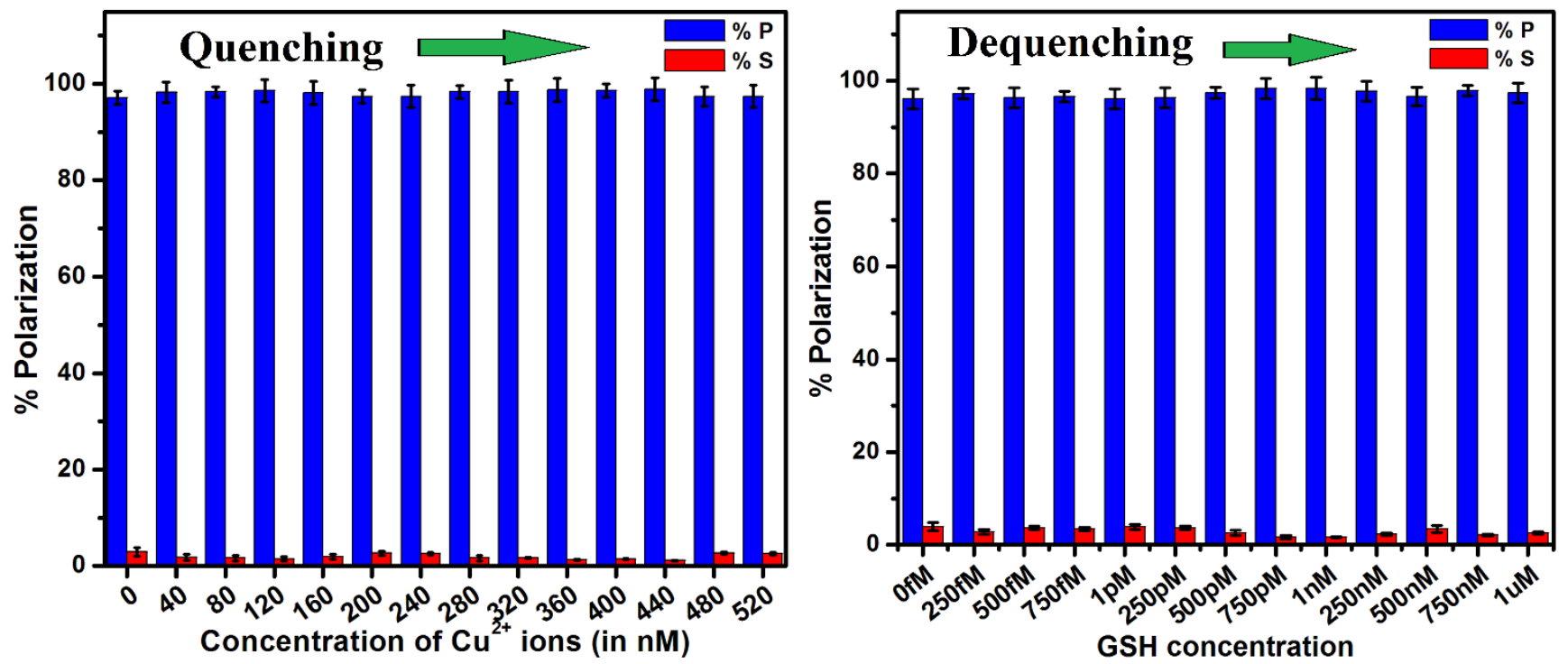

Figure S10. Polarized emission from ARS upon quenching with different $\mathrm{Cu}^{2+}$ ions concentrations (left).

Polarized emission from ARS upon dequenching with different GSH concentrations (right). 